\title{
Structure-Activity Relationship for Fe(III)-Salen-Like Complexes as Potent Anticancer Agents
}

\author{
Zahra Ghanbari, ${ }^{1}$ Mohammad R. Housaindokht, ${ }^{2}$ Mohammad Izadyar, ${ }^{1}$ \\ Mohammad R. Bozorgmehr, ${ }^{3}$ Hossein Eshtiagh-Hosseini, ${ }^{2}$ Ahmad R. Bahrami, \\ Maryam M. Matin, ${ }^{2}$ and Maliheh Javan Khoshkholgh ${ }^{1}$ \\ ${ }^{1}$ Department of Chemistry, Faculty of Science, Ferdowsi University of Mashhad, Mashhad 9177948974, Iran \\ ${ }^{2}$ Institute of Biotechnology, Ferdowsi University of Mashhad, Mashhad 9177948974, Iran \\ ${ }^{3}$ Department of Chemistry, Mashhad Branch, Islamic Azad University, Mashhad 9187147578, Iran
}

Correspondence should be addressed to Mohammad R. Housaindokht; housain@um.ac.ir and

Hossein Eshtiagh-Hosseini; heshtiagh@um.ac.ir

Received 8 January 2014; Accepted 19 February 2014; Published 6 April 2014

Academic Editors: E. R. Dockal, B. I. Kharisov, and A. I. Matesanz

Copyright (C) 2014 Zahra Ghanbari et al. This is an open access article distributed under the Creative Commons Attribution License, which permits unrestricted use, distribution, and reproduction in any medium, provided the original work is properly cited.

\begin{abstract}
Quantitative structure activity relationship (QSAR) for the anticancer activity of Fe(III)-salen and salen-like complexes was studied. The methods of density function theory (B3LYP/LANL2DZ) were used to optimize the structures. A pool of descriptors was calculated: 1497 theoretical descriptors and quantum-chemical parameters, shielding NMR, and electronic descriptors. The study of structure and activity relationship was performed with multiple linear regression (MLR) and artificial neural network (ANN). In nonlinear method, the adaptive neuro-fuzzy inference system (ANFIS) was applied in order to choose the most effective descriptors. The ANN-ANFIS model with high statistical significance $\left(R_{\text {train }}^{2}=0.99, \mathrm{RMSE}=0.138\right.$, and $\left.Q^{2}{ }_{\text {LOO }}=0.82\right)$ has better capability to predict the anticancer activity of the new compounds series of this family. Based on this study, anticancer activity of this compound is mainly dependent on the geometrical parameters, position, and the nature of the substituent of salen ligand.
\end{abstract}

\section{Introduction}

Despite several efforts in the treatment of cancer, because of several limitations that using medications has, this disease became a big problem for the health of societies. The purpose is to develop medications with more anticancer activity and less toxicity than the present medications. Metallic compounds have been widely studied due to their major role in biological activities. Since the introduction of cisplatin as anticancer medication, a comprehensive study has been performed on the metal complexes and the medicinal features of these compounds [1-3]. Currently, metal complexes of different transition metal are preferred candidates for the treatment of different sort of cancer. Medicinal inorganic chemistry can employ different strategies in the development of unique properties of metal ions for design of new anticancer drugs. However design, synthesis, and structural characterization of metallodrugs have attracted a lot of interest due to their applications in anticancer fields. The performance of these compounds is explained on the basis of many mechanisms including intercalation, inhibition of DNA and RNA [4-8]. Lipinski, Murcke, and coworkers had an important role in concentrating on the importance of features of such medication on the basis of its shape. The anticancer activity of the metallic complexes has also an adjacent relationship with the type of central metal and binding ligand to it $[1$, 9]. Meanwhile, bis(salicylidene)ethylenediamine ligands are subject of the study for a long time and several of them including various metals have been synthesized [9-26]. Ironsalen complexes have been studied since 1931 because of the physical and biological features and it has been cleared that salen complexes of F(III) have anticancer features on the MCF7 cells [27]. Metallosalens damage DNA/RNA in vitro. Iron-salen derivatives produce hydroxyl radicals in the 
presence of reducing agent of dithiothreitol (DTT) and damage DNA $[28,29]$. The probability of discovering a natural medication which did not need correction or probability of providing a defined compound as a medication is too rare. Today, the molecular and chemical computing models are used in designing new medications which resulted in saving time and cost and designing medications with more potential. Among various computational methods, QSAR has a remarkable role in designing a medication. In fact, underlying basis of SAR (structure-activity relationships) focuses on the elucidation of structure and biological effects but QSAR attempts to form a quantitative relationship between them [30-33]. QSAR models are mathematical equations which relate the chemical structure of compounds to their biological activity [34-37]. There are theoretical pathways which are used to encode the information of the molecular structure into numbers to acquire these equations $[38,39]$. The relation of construction activity of anticancer Fe(III)salen-like complexes had been studied a lot in the past. One important viewpoint of the researchers in the past works showed that the nature of substitutes and bridge between $\mathrm{D}$-amino groups play the main role in defining the anticancer feature of these compounds. This happens in a way that increases in the aromaticity of $\mathrm{D}$-amino bridges and replacing of hydroxyl groups with methoxy (except some items) will lead to increase in anticancer feature [2, 28]. But, considering available information resources, there is no report, in quantities viewpoint, QSAR, to show this relation more exactly and with more details.

In this paper, what we considered has been searching for QSAR relation for Fe(III)-salen and salen-like complexes with linear and nonlinear methods and designing a model with high statistical significance to predict anticancer activity of new compounds series of this family. QSAR analysis in this study is based on the mathematical relationship between biological activity and structural geometric, quantum-chemical, electronic, and spectral features. For this purpose, substitutes on salen ligand and chloride ligand replacement with $\mathrm{N}$ heterocyclic ligands were studied. With this replacement, anticancer activity will be changed in a wide range and modeling is a tool used to understand and predict diverse activities. Two main goals were pursued in this work: (1) the study of the influence of the increase in aromatic rings on the bridge between $\mathrm{D}$-amino groups on the anticancer activity and (2) the study of the influence of the change in the position and nature of the salen ligand on the anticancer activity. The resulting equations adequately describe the biological activity of these complexes and assign important descriptors of compounds for efficient anticancer activity.

\section{Materials and Methods}

2.1. Data Set. Biological data which were used in this work were the anticancer activity of $26, \mathrm{Fe}$ (III)-salen and salenlike complexes against human breast cancer cell line (MCF7) in terms of $\mathrm{IC}_{50}$. The quantities of $\mathrm{IC}_{50}$, under the same conditions, were collected from previous studies [2, 27, 28]. The structures of studied compounds and quantities of their anticancer activity have been reported in Figure 1 and Table 1, respectively. The activity data have been converted into logarithm units $\left(\mathrm{PIC}_{50}\right)$ then were used for modeling.

2.2. Geometry Optimization and Molecular Descriptors Calculation. The optimized 3D geometry of the molecules was achieved using Gaussian 03 software as well as B3LYP technique and LANL2DZ basis set. This method presents satisfactory results for the optimization of the 3D geometry of the metal complexes [40, 41]. Dragon packages, Gaussian 03, and AIM were used for calculation of molecular descriptors $[41,42]$. A pool of descriptors was calculated by Dragon software for each molecule including parameters of all types such as constitutional, topological, geometrical, GETAWAY, WHIM, 3D-MoRSE, Molecular Walk Counts, BCUT descriptors, 2D autocorrelations, aromaticity indices, randic molecular profiles, radial distribution functions, functional groups, atom-centered fragments, empirical and properties [42]. In addition, highest occupied molecular orbital (HOMO), lowest unoccupied molecular orbital (LUMO), dipole moment, natural charge, shielding NMR, and total energy were calculated by DFT method. Chemical hardness $(\eta)$, chemical softness $(\sigma)$, chemical potential $(\mu)$, and electrophilicity $(\omega)$ were calculated according to the equations [38, 43]. Charge density $(\rho(r))$ and Laplacian of the electronic charge density $\nabla^{2} \rho(r)$ which were calculated using AIM software were based on the quantum theory of atoms in molecules [44].

2.3. Descriptor Selection. Computed descriptors and empirical data were analyzed using SPSS software [45]. These data were put in a quadratic matrix in which its order is equal to the number of molecules and descriptors. Among descriptors, those which had more correlation with anticancer activity were saved and the others were omitted. In recent years finding the most efficient descriptors from a pool of variables plays a fundamental role in QSAR studies [46, 47]. In this work, finding the most efficient descriptors was performed with linear and nonlinear methods; finally, created models were compared. Selection of the most efficient attributes and obtaining of the final equation are very convenient by linear techniques. In this work, QSAR equations between independent descriptors and $\mathrm{PIC}_{50}$ empirical parameters were obtained as a response in a way that each category of descriptors was considered individually and the most efficient descriptors by multiple linear regression- (MLR-) stepwise were selected. Since in the nonlinear method ANN cannot select the most significant descriptors, ANFIS algorithm, a developed algorithm based on neural network and fuzzy logic, was used. This algorithm can characterize extremely nonlinear functions. ANFIS was used here for investigation; the most effective parameters in a target function and the most effective descriptors were selected [48].

2.4. Model Development. In this work MLR was employed as linear technique and ANN as nonlinear ones for the QSAR models.

2.4.1. MLR-Stepwise. In this stage study of structure and activity relationship was performed with multiple linear 


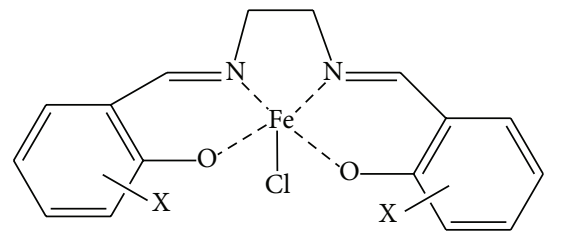

$\begin{array}{cc}\mathrm{X}= & \text { Compound } \\ \mathrm{H} & 1 \\ 3,3-\mathrm{OH} & 2 \\ 4,4-\mathrm{OH} & 3 \\ 5,5-\mathrm{OH} & 4 \\ 3,3-\mathrm{OMe} & 5 \\ 4,4-\mathrm{OMe} & 6 \\ 5,5-\mathrm{OMe} & 7\end{array}$

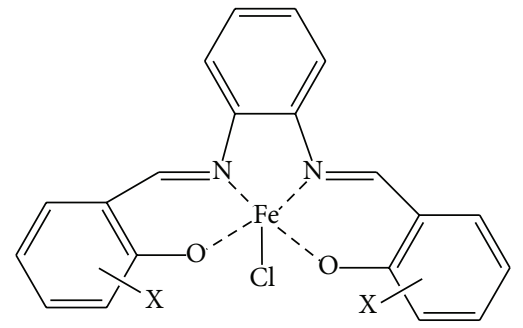

$\begin{array}{cc}\mathrm{H} & 8 \\ 3,3-\mathrm{OH} & 9 \\ 4,4-\mathrm{OH} & 10 \\ 5,5-\mathrm{OH} & 11 \\ 3,3-\mathrm{OMe} & 12 \\ 4,4-\mathrm{OMe} & 13 \\ 5,5-\mathrm{OMe} & 14\end{array}$

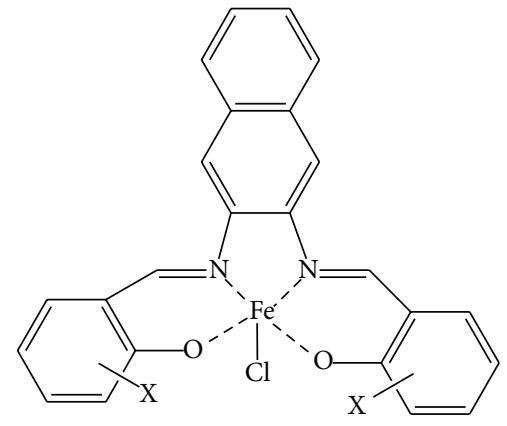

$\mathrm{H} \quad 15$

3,3-OH 16

$4,4-\mathrm{OH}$

$5,5-\mathrm{OH}$

3,3-OMe 19

4,4-OMe 20

$5,5-\mathrm{OMe}$<smiles></smiles>

1H-imidazole(Himz)

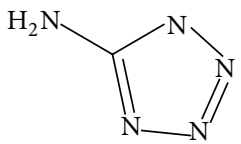

1H-tetrazol-5-amine(Hatz)

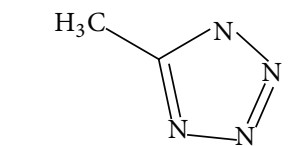

5-methyle-1H-tetrazol(Hmtz)

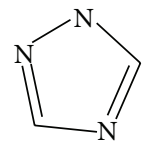

1H-1,2,4-triazol(Htriz)

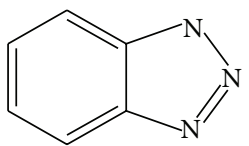

1H-benzotriazol(Hbtriz)

FIGURE 1: Structure of the complexes studied. 
TABLE 1: Experimental and predicted values of PIC $_{50}$ for various salen complexes by MLR and ANN models.

\begin{tabular}{|c|c|c|c|}
\hline \multirow{2}{*}{ Structure } & \multirow{2}{*}{$\mathrm{PIC}_{50}$ experimental } & \multicolumn{2}{|c|}{$\mathrm{PIC}_{50}$ predicted } \\
\hline & & ANN & MLR \\
\hline 1 & -1.34227 & -1.30399 & -1.34220 \\
\hline 2 & -1.77815 & -1.81275 & -1.77810 \\
\hline 3 & -1.77815 & -2.15957 & -1.77810 \\
\hline 4 & -1.77815 & -2.11785 & -1.77810 \\
\hline 5 & -1.77815 & -1.47180 & -1.77820 \\
\hline 6 & -1.07918 & -0.82961 & -1.07920 \\
\hline 7 & -0.65321 & -0.54961 & -0.65320 \\
\hline 8 & -0.11394 & -0.54747 & -0.11390 \\
\hline 9 & 0.52288 & 0.24813 & 0.60580 \\
\hline 10 & -1.77815 & -1.80770 & -1.78810 \\
\hline 11 & -1.77815 & -1.35897 & -1.78810 \\
\hline 12 & -0.61278 & -0.67836 & -0.61280 \\
\hline 13 & 0.1549 & -0.01870 & 0.15500 \\
\hline 14 & -0.49136 & -0.46266 & -0.12430 \\
\hline 15 & 0.30103 & -0.24032 & 0.30110 \\
\hline 16 & 0.69897 & 0.73866 & 0.69890 \\
\hline 17 & -2.00000 & -1.12536 & -1.60450 \\
\hline 18 & -2.00000 & -1.14808 & -2.00000 \\
\hline 19 & -0.17609 & -0.22396 & -0.17610 \\
\hline 20 & 0.30103 & 0.41782 & 0.30100 \\
\hline 21 & -0.11394 & -0.21354 & -0.11390 \\
\hline 22 & -1.37475 & -1.42517 & -1.37470 \\
\hline 23 & -1.11727 & -1.45753 & -1.11730 \\
\hline 24 & -1.31387 & -1.10417 & -1.31390 \\
\hline 25 & -1.26245 & -1.61461 & -1.71060 \\
\hline 26 & -1.22789 & -1.04990 & -1.22790 \\
\hline Cisplatin & -1.25527 & & \\
\hline
\end{tabular}

$\mathrm{PIC}_{50}=-\log \left(\mathrm{IC}_{50}\right)$.

TABLE 2: Descriptors used in MLR.

\begin{tabular}{|c|c|c|c|}
\hline Number & Symbol & Chemical meaning & Type \\
\hline 1 & MATS8e & $\begin{array}{l}\text { Moreau autocorrelation-lag8/weighted by atomic sanderson } \\
\text { electronegativities }\end{array}$ & $2 \mathrm{D}$ autocorrelation \\
\hline 2 & Mor28u & Signal 28/unweighted & 3D-MoRSE \\
\hline 3 & $\mathrm{H} 8 \mathrm{~m}$ & $\mathrm{H}$ autocorrelation of lag8/weighted by atomic masses & GETAWAY \\
\hline 4 & $\mathrm{CICl}$ & $\begin{array}{l}\text { Complementary information content } \\
\text { (neighborhood symmetry of 1-order) }\end{array}$ & Topological \\
\hline 5 & G3s & $\begin{array}{l}\text { 3st component symmetry directional WHIM index/ } \\
\text { weighted by atomic electrotopological states }\end{array}$ & WHIM \\
\hline
\end{tabular}

regression (MLR) in SPSS software $[49,50]$. The most efficient descriptors by multiple linear regression- (MLR-) stepwise were selected. Then five descriptors were selected by this procedure [51, 52]. Table 2 represents the selected variables and their chemical meanings. The correlation matrix among these descriptors is shown in Table 3. Find correlation between $\mathrm{PIC}_{50}$ and five descriptors is given by

$$
\begin{aligned}
\mathrm{PIC}_{50}= & -8.991+1.354 \mathrm{CIC} 1+11.336 \mathrm{H} 8 \mathrm{~m} \\
& -2.045 \mathrm{MATS} 8 \mathrm{e}+22.943 \mathrm{G} 3 \mathrm{~s}-2.032 \text { Mor28u. }
\end{aligned}
$$

2.4.2. ANN-ANFIS. Artificial neural networks are generally used for nonlinear regressions $[53,54]$. The most effective descriptors were selected by using ANFIS algorithm. For comparing linear and nonlinear selection in Table 4, the most efficient descriptors from the pool of descriptors which are selected by ANFIS models and their chemical meanings have been represented. In this study multilayer feed-forward (MLFF) network with back-propagation (BP) learning was employed and its overview is shown in Figure 2. We use the Matlab 7.0 program in these calculations $[48,55]$. For training 
of neural network, obtained descriptor and anticancer activity were used as inputs and outputs, respectively. After the training of the network, the resulting ANN model was used to predict the activity of the test set compounds. Normalized inputs and outputs have better effect on training. The network includes some hidden layers with sigmoid neurons and final linear layer. The function of nonlinear transmit to the network provides the ability of learning linear and nonlinear relationship between inputs and outputs and the external linear layer enables the outputs to be out of the range of -1 and +1 . With performing the network, the statistical weights of each of the descriptors will change alternatively till the error between anticipated values of $\mathrm{PIC}_{50}$ and the values of empirical PIC $_{50}$ (target vector) is minimized. Several models with various numbers of hidden layers and neurons were designed and they are optimized by a systematic search method. The best network model with 3 layers and 9, 8 and 1 neuron was selected. The network was trained with Levenberg-Marquardt (LM) algorithm [56, 57].

2.4.3. Validation of QSAR Models. Validation process is a necessary step in QSAR. In fact the QSAR models were validated by the calculation of the statistical terms (correlation coefficient $R^{2}$, cross-validation $Q^{2}$, standard error of prediction $S$, root mean square error RMSE, etc.). Crossvalidation is the statistical method of partitioning a sample of data into training set and test set. The test set was used for external validation. One of the cross-validation methods is LOO where one object at a time is removed from the data set and then predicted by generated model. The $Q^{2}$ LOO was calculated using

$$
Q^{2}=\frac{1-\text { Press }}{\text { SSY }}
$$

where Press $=\sum\left(Y_{\text {pred }}-Y_{\text {actual }}\right)^{2}$ and SSY $=$ $\sum\left(Y_{\text {actual }}-Y_{\text {mean }}\right)^{2}$ and where $Y_{\text {pred }}$ is a predicted value of activity, $Y_{\text {actual }}$ is an actual or experimental value of activity, and $Y_{\text {mean }}$ is the mean activity value [38]. We apply cross-validation method to determine that QSAR models have ability to correctly predict the biological activities of new compounds. Results have been reported in Table 5.

\section{Results and Discussion}

The underlying basis of this study has focused on elucidation of the molecular structure and anticancer activity of these compounds with two methods of MLR-stepwise and ANNANFIS. Here QSAR studies were confident to receptorindependent (RI) QSAR analyses and the geometry of the receptor is neglected [35]. The data set was divided into training and test sets. The test set was used for external validation. The ANN-ANFIS model with high statistical significance has better capability to predict anticancer activity of new compounds series of this family (Figure 3 ).

The QSAR models should be interpretable and it is important to explain the selected descriptors [58]. Definition of each selected descriptor was presented here. Anticancer activity of this series of compounds could not be attributed to one or two structural features of the molecules and the anticancer activity is the product of optimizing a collection of descriptors. It has been observed that in MLR-stepwise model 2D autocorrelation, 3D-MoRSE, GETAWAY, topological, and WHIM descriptors have more effect on anticancer activity than quantum chemical ones. The values of the mean effect (MF) were calculated according to (3) to indicate the relative importance of these descriptors. Consider

$$
\mathrm{MF}_{j}=\frac{\beta_{j} \sum_{i=1}^{i=n} d_{i j}}{\sum_{j}^{m} \beta_{j} \sum_{i}^{n} d_{i j}},
$$

where $\mathrm{MF}_{j}$ represents the mean effect of the considered descriptor $j, b_{j}$ is the coefficient of the descriptor $j, d_{i j}$ stands for the value of the target descriptors for each molecule, and, eventually, $m$ is the descriptors number in the model $[46,59]$.

MF values are $0.506,0.415,0.048$, and $0.030,0$ for G3s, CIC1, H8m, Mor28u, and MATS8e, respectively. The high value of mean effect for G3s shows the significance of this descriptor in the model. G3s is one of the global WHIM descriptors which display a positive sign on $\mathrm{PIC}_{50}$. Weighted holistic invariant molecular (WHIM) descriptors are geometrical descriptors which show molecular 3D information regarding molecular size, shape, symmetry, and atom distribution. WHIM descriptors are suitable for complex properties. In G3s, WHIM weighted covariance matrixes were provided by the electrotopological state indexes of Kier and Hall [38]. CICl (complementary information content with neighborhood symmetry of 1-order) is the second order of importance. It is a topological descriptor. Topological indexes are single indexes derived from a molecular graph which can be sensitive to one or more structural features of the molecule such as size, shape, symmetry, branching, and cyclicity. This descriptor shows the molecular symmetry by measuring the neighborhood of the atoms (through the value of the vertex degrees) located at a first-order distance (one single bond) of a considered atom, for each vertex in $G$ $[38,60]$. MATS8e (Moreau autocorrelation-lag8/weighted by atomic Sanderson electronegativities) is one of the $2 \mathrm{D}$ autocorrelation descriptors by Broto-Moreau calculated from the molecular graph by summing the products of atom weights of the terminal atoms of all the paths of length 8 , using the Sanderson electronegativities as weighting scheme (the lag). Variation in toxicity as a function of position and nature of the substituent is determined by $2 \mathrm{D}$ parameters. It shows that replacing hydroxyl group with methoxy in different positions of the salen ligand plays a crucial role regarding toxicity. It is well known that the stereo chemical moieties of the investigated compounds could affect biological activity so 2D models of molecules can provide stereo chemical information [38, 61]. H8m ( $\mathrm{H}$ autocorrelation of lag8/weighted by atomic masses) is of the GETAWAY descriptors which are geometrical descriptors which encode information on the effective position of substituents and fragments in the molecular space. In fact GETAWAY descriptors encode both the geometrical information given by the influence molecular matrix and the topological information given by the molecular graph [38, 62]. Mor28u (Signal 28/unweighted) is one of the 3D-MoRSE descriptors which represent structures based 
TABLE 3: Correlation coefficient matrix of the selected descriptors.

\begin{tabular}{lccccc}
\hline & MATS8e & Mor28u & H8m & CIC1 & G3s \\
\hline MATS8e & 1 & -0.273 & 0.270 & 0.026 & -0.283 \\
Mor28u & & 1 & -0.109 & -0.668 & -0.347 \\
H8m & & & 1 & -0.124 & 1 \\
CIC1 & & & & -0.259 \\
G3s & & & & 1 \\
\hline
\end{tabular}

TABLE 4: Five most efficient descriptors selected by ANFIS models.

\begin{tabular}{lclc}
\hline No. & Symbol & Chemical meaning & Type \\
\hline 1 & Mor28p & Signal28/weighted by atomic polarizabilities & 3D-MoRSE \\
2 & SPI & Superpendentic index & Topological \\
3 & RDF110m & Radial distribution function 11.0/weighted by atomic masses & RDF \\
4 & SPCN8 & Shielding NMR $(\mathrm{ppm})$ of Nitrogen8 & NMR \\
5 & MATS5v & Moreau autocorrelation-lag5/weighted by atomic van der Waals & 2D autocorrelation \\
\hline
\end{tabular}

TABLE 5: Results and validation of QSAR models.

\begin{tabular}{lccc}
\hline & $R_{\text {train }}^{2}$ & $Q^{2}{ }_{\text {LOO }}$ & RMSE \\
\hline MLR-stepwise & 0.863 & 0.769 & 0.342 \\
ANN-ANFIS & 0.999 & 0.820 & 0.138 \\
\hline
\end{tabular}

on electron diffraction descriptors so they can reveal the skeleton and substituents information for a molecule. Various physicochemical properties such as atomic mass, partial atomic charges, and atomic polarizability were considered to present high flexibility of a molecule. The form of the intensity distribution $I(s)$ is given by

$$
I(s)=\sum_{i=2}^{N} \sum_{j=1}^{i-1} A_{i} A_{j} \frac{\sin s r_{i j}}{s r_{i j}}, \quad s=0, \ldots, 31.0 \AA^{-1},
$$

where $N$ is the number of atoms, $r_{i j}$ is the distance between atoms $i$ and $j, A_{i}$ can be any atomic property of atom $i$ such as atomic number, mass, partial atomic charge, or atomic polarizability, and $s$ is a reciprocal distance. The value of $s$ was considered only at discrete positions within a certain range, between 0 and $31 \AA^{-1}$. For Mor28u, an atomic mass weighted scheme was used and $s$ was equal to $27 \AA^{-1}[63,64]$.

Two QSAR models were built here using various types of descriptors. In nonlinear model Mor28p, Signal28/weighted by atomic polarizabilities is one of the 3D-MORSE descriptors whose autocorrelation vectors are weighted by atom polarizabilities. SPI (superpendentic index) is one of the topological descriptors which derived from the $H$-depleted molecular graph and is calculated according to the following:

$$
\int_{p}=\left(\sum_{i=1}^{A} \pi_{m} d_{i m}\right)^{1 / 2}
$$

where $d$ is the topological distances, that is, row of the pendent matrix, and $m$ is the number of terminal vertices, that is, the column of the pendent matrix $[65,66]$. RDF110m is one of the 3D-radial distribution function (RDF) descriptors which were proposed based on a radial distribution function. The radial distribution function is probability distribution to find an atom in a spherical volume of radius $R$. RDF descriptors are independent of the size and rotation of the entire molecule. They describe the steric hindrance or the structure/activity properties of a molecule. The general equation of the radial distribution function is in accordance with the following:

$$
g(R)=f \cdot \sum_{i=1}^{A-1} \sum_{j=i+1}^{A} w_{i} \cdot w_{j} \cdot e^{-\beta \cdot\left(R-r_{i j}\right)^{2}}
$$

where $f$ is a scaling factor, $w$ is the characteristic atomic properties of the atoms $i$ and $j, r_{i j}$ is the interatomic distance between the $i$ th and $j$ th atom, and $A$ is the number of atoms. The exponential term contains the distance $r_{i j}$ between the atoms $i$ and $j$ and the smoothing parameter $\beta$ that defines the probability distribution of the individual interatomic distances. $\beta$ can be interpreted as a temperature factor which defines the movement of atoms. The RDF descriptor provides valuable information about the bond distances, ring types, planar and nonplanar systems, and atom types $[38,59]$. SPCN8 is shielding NMR (ppm) of the nitrogen 8 which is calculated by Gaussian 03. Final MATS5v (Moran autocorrelation-lag 5/weighted by atomic Sanderson electronegativities) is one of the $2 \mathrm{D}$-autocorrelation descriptors.

This study provides deeper insight into the antitumor activity of the Fe(III)-salen-like complexes. Based on the above discussion the anticancer activity of this compound is mainly dependent on the geometrical parameters and position and nature of the substituent of the salen ligand. Data analysis shows that the increase in aromatic rings on the bridge between $\mathrm{D}$-amino groups causes more activity of the complex. Geometrical parameters are important in the ligand transportation through the cell membrane. Also change in the position of the substituent of the salen ligand 


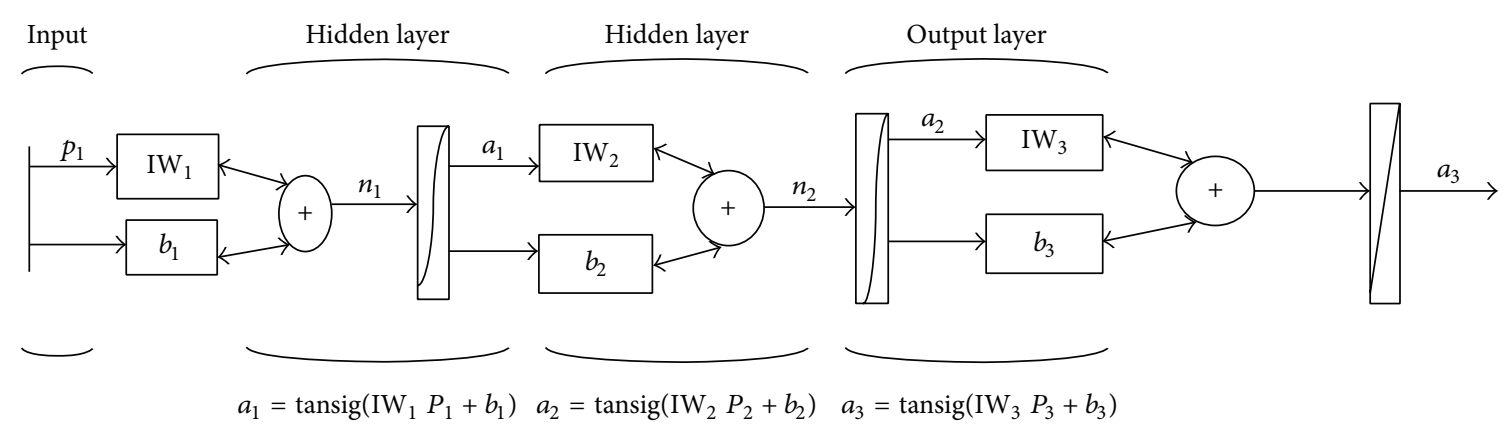

FIGURE 2: The architecture of feedforward neural network.

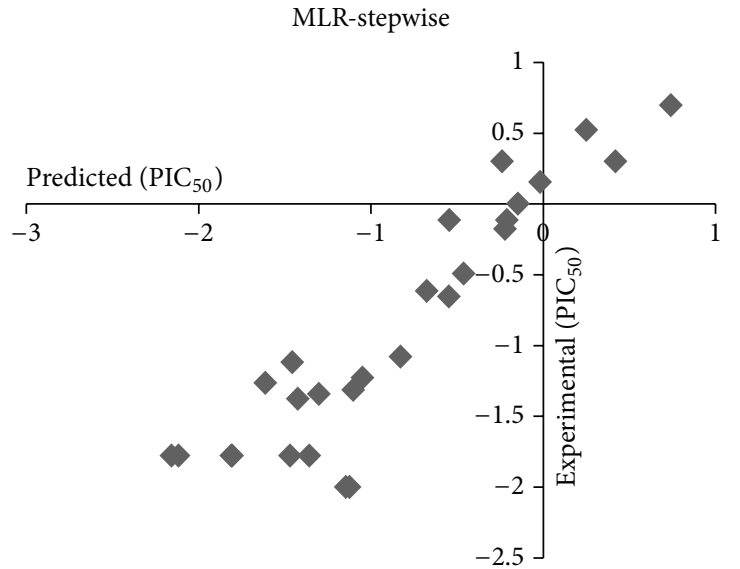

(a)

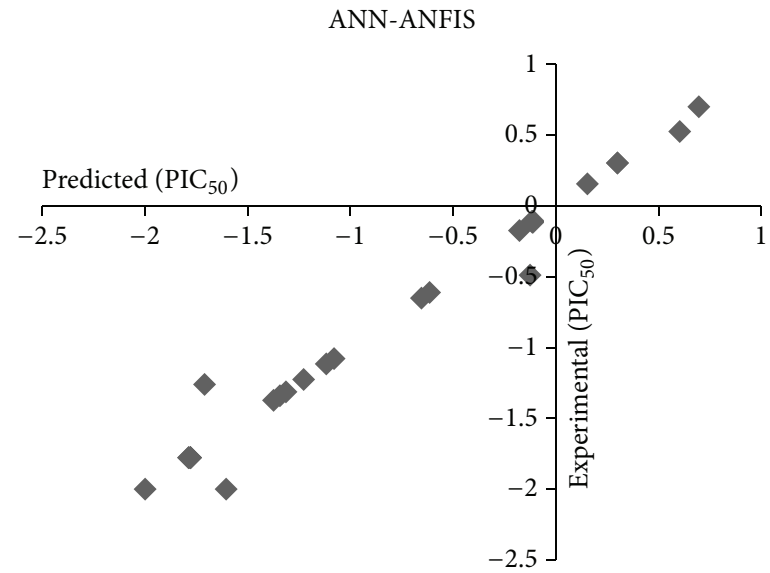

(b)

FIGURE 3: Results and comparison of MLR-stepwise and ANN-ANFIS models.

changes the anticancer activity. The nature of the substituent has a sharp effect on the biological activity. Our studies on the influence of $\mathrm{Cl}$ ligand replacing the heterocyclic $\mathrm{N}$ donor ligands show that $1 \mathrm{H}$-tetrazol-5-amin(Hatz) increases in activity. Results show that change of $\mathrm{Cl}$ ligand on the heterocyclic $\mathrm{N}$-donor ligands has a minor effect compared to aromatic group replacement on the anticancer activity which is shown in Figure 4.

\section{Conclusion}

Some of iron(III)-salen complexes have a very desirable anticancer activity against MCF7 cells. Their anticancer activity is the result of optimizing a collection of descriptors, considering that acquired results could not attribute the anticancer activity to one or two special structural features. Also, the results of this study show the high ability of nonlinear methods which resulted from fuzzy logic and neural network in anticipating the anticancer activity of new series of salen complexes such as iron(III). The ANN-ANFIS model with high statistical significance has better capability to predict anticancer activity of the new compounds series of this family. The results show the importance of the geometrical parameters and position and nature of the substituent of the salen ligand on the anticancer activity.

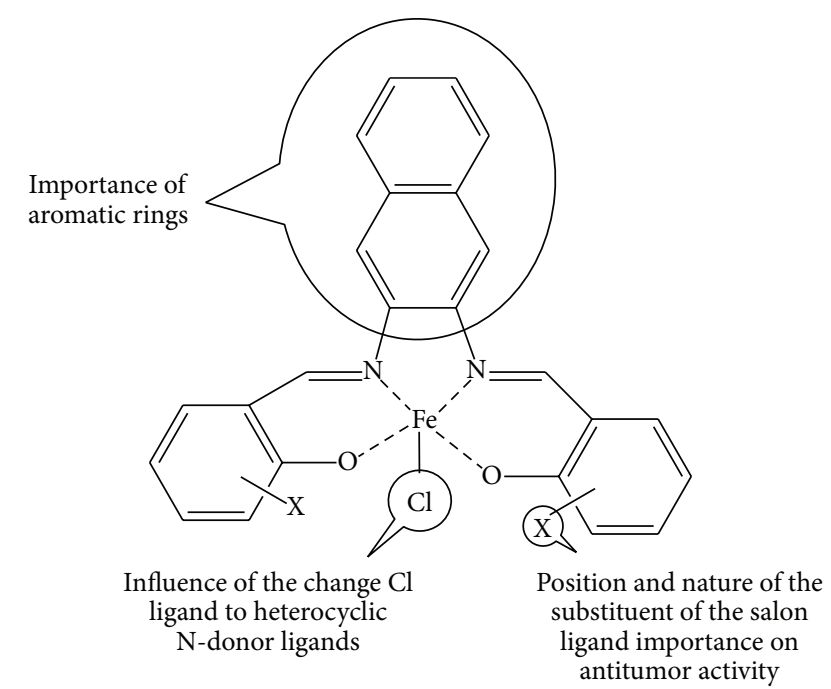

FIGURE 4: Importance of the position and nature of the substituent of the salen ligand.

\section{Conflict of Interests}

The authors declare that there is no conflict of interests regarding the publication of this paper. 


\section{Acknowledgment}

Financial support of the Ferdowsi University of Mashhad, Iran (P/176-89/3/23 \& 3/19728-90/10/13), is gratefully acknowledged.

\section{References}

[1] M. E. Bravo-Gómez, J. C. García-Ramos, I. Gracia-Mora, and L. Ruiz-Azuara, "Antiproliferative activity and QSAR study of copper(II) mixed chelate $\left[\mathrm{Cu}(\mathrm{N}-\mathrm{N})(\right.$ acetylacetonato) $] \mathrm{NO}_{3}$ and $[\mathrm{Cu}(\mathrm{N}-\mathrm{N})($ glycinato $)] \mathrm{NO}_{3}$ complexes," Journal of Inorganic Biochemistry, vol. 103, no. 2, pp. 299-309, 2009.

[2] K. I. Ansari, S. Kasiri, J. D. Grant, and S. S. Mandal, "Fe(III)salen and salphen complexes induce caspase activation and apoptosis in human cells," Journal of Biomolecular Screening, vol. 16, pp. 26-35, 2010.

[3] N.-U. H. Khan, N. Pandya, R. I. Kureshy et al., "Synthesis, characterization, DNA binding and cleavage studies of chiral $\mathrm{Ru}(\mathrm{II})$ salen complexes," Spectrochimica Acta A: Molecular and Biomolecular Spectroscopy, vol. 74, no. 1, pp. 113-119, 2009.

[4] N. Zhang, Y. h. Fan, and Z. Zhang, "Syntheses, crystal structures and anticancer activities of three novel transition metal complexes with Schiff base derived from 2-acetylpyridine and 1tryptophan," Inorganic Chemistry Communications, vol. 22, pp. 68-72, 2012.

[5] I. Ali, W. A. Wani, K. Saleem, and M. F. Hseih, "Design and synthesis of thalidomide based dithiocarbamate $\mathrm{Cu}(\mathrm{II}), \mathrm{Ni}(\mathrm{II})$ and $\mathrm{Ru}(\mathrm{III})$ complexes as anticancer agents," Polyhedron, vol. 56, pp. 134-143, 2013.

[6] V. D. Reddy, D. Dayal, D. J. Szalda, S. C. Cosenza, and M. V. Ramana Reddy, "Syntheses, structures, and anticancer activity of novel organometallic ruthenium-maltol complexes," Journal of Organometallic Chemistry, vol. 700, pp. 180-187, 2012.

[7] Z. Afrasiabi, E. Sinn, S. Padhye et al., "Transition metal complexes of phenanthrenequinone thiosemicarbazone as potential anticancer agents: synthesis, structure, spectroscopy, electrochemistry and in vitro anticancer activity against human breast cancer cell-line, T47D," Journal of Inorganic Biochemistry, vol. 95, no. 4, pp. 306-314, 2003.

[8] P. C. Bruijnincx and P. J. Sadler, "New trends for metal complexes with anticancer activity," Current Opinion in Chemical Biology, vol. 12, no. 2, pp. 197-206, 2008.

[9] W. L. Jorgensen, "The many roles of computation in drug discovery," Science, vol. 303, no. 5665, pp. 1813-1818, 2004.

[10] H. Pellissier, "Recent developments in asymmetric cyclopropanation," Tetrahedron, vol. 64, no. 30-31, pp. 7041-7095, 2008.

[11] R. Luna-García, B. M. Damián-Murillo, V. Barba, H. Höpfl, H. I. Beltrán, and L. S. Zamudio-Rivera, "Structure and conformational motion of seven-coordinate diorganotin(IV) complexes derived from salen and salan type ligands," Journal of Organometallic Chemistry, vol. 694, no. 24, pp. 3965-3972, 2009.

[12] G. Barone, A. Ruggirello, A. Silvestri, A. Terenzi, and V. T. Liveri, "Fluorescence emission and enhanced photochemical stability of $\mathrm{Zn}^{\mathrm{II}}$-5-triethyl ammonium methyl salicylidene orthophenylendiiminate interacting with native DNA," Journal of Inorganic Biochemistry, vol. 104, no. 7, pp. 765-773, 2010.

[13] S. Routier, J.-L. Bernier, J.-P. Catteau, and C. Bailly, "Recognition and cleavage of DNA by a distamycin-salen-copper conjugate," Bioorganic and Medicinal Chemistry Letters, vol. 7, no. 13, pp. 1729-1732, 1997.
[14] G. Verquin, G. Fontaine, E. Abi-Aad, E. Zhilinskaya, A. Aboukaïs, and J.-L. Bernier, "EPR study of copper(II) complexes of hydroxysalen derivatives in order to be used in the DNA cleavage," Journal of Photochemistry and Photobiology B: Biology, vol. 86, no. 3, pp. 272-278, 2007.

[15] T. W. Failes and T. W. Hambley, "Towards bioreductively activated prodrugs: $\mathrm{Fe}$ (III) complexes of hydroxamic acids and the MMP inhibitor marimastat," Journal of Inorganic Biochemistry, vol. 101, no. 3, pp. 396-403, 2007.

[16] G. A. Woldemariam and S. S. Mandal, "Iron(III)-salen damages DNA and induces apoptosis in human cell via mitochondrial pathway," Journal of Inorganic Biochemistry, vol. 102, no. 4, pp. 740-747, 2008.

[17] A. Meshkini and R. Yazdanparast, "Chemosensitization of human leukemia K562 cells to taxol by a Vanadium-salen complex," Experimental and Molecular Pathology, vol. 89, no. 3, pp. 334-342, 2010.

[18] M. Mohammadi and R. Yazdanparast, "Methoxy VO-salen complex: in vitro antioxidant activity, cytotoxicity evaluation and protective effect on $\mathrm{CCl}_{4}$-induced oxidative stress in rats," Food and Chemical Toxicology, vol. 47, no. 4, pp. 716-721, 2009.

[19] F. Fucassi, J. E. Lowe, K. D. Pavey et al., " $\alpha$-Lipoic acid and glutathione protect against the prooxidant activity of SOD/catalase mimetic manganese salen derivatives," Journal of Inorganic Biochemistry, vol. 101, no. 2, pp. 225-232, 2007.

[20] K. I. Ansari, J. D. Grant, S. Kasiri, G. Woldemariam, B. Shrestha, and S. S. Mandal, "Manganese(III)-salens induce tumor selective apoptosis in human cells," Journal of Inorganic Biochemistry, vol. 103, no. 5, pp. 818-826, 2009.

[21] N. Beeharry, J. E. Lowe, A. R. Hernandez et al., "Linoleic acid and antioxidants protect against DNA damage and apoptosis induced by palmitic acid," Mutation Research. Fundamental and Molecular Mechanisms of Mutagenesis, vol. 530, no. 1-2, pp. 2733, 2003.

[22] J. Fonseca, J. Martinez, L. Cunha-Silva, A. L. Magalhães, M. T. Duarte, and C. Freire, "Insights into electronic and structural properties of novel $\mathrm{Pd}(\mathrm{II})$ salen-type complexes," Inorganica Chimica Acta, vol. 363, no. 14, pp. 4096-4107, 2010.

[23] Y. Fan, W. You, W. Huang, J.-L. Liu, and Y.-N. Wang, "Salen-type nickel(II), palladium(II) and copper(II) complexes having chiral and racemic camphoric diamine components," Polyhedron, vol. 29, no. 3, pp. 1149-1155, 2010.

[24] J. G. Muller, L. A. Kayser, S. J. Paikoff et al., "Formation of DNA adducts using nickel(II) complexes of redox-active ligands: a comparison of salen and peptide complexes," Coordination Chemistry Reviews, vol. 185-186, pp. 761-774, 1999.

[25] Q. Jiang, N. Xiao, P. Shi, Y. Zhu, and Z. Guo, "Design of artificial metallonucleases with oxidative mechanism," Coordination Chemistry Reviews, vol. 251, no. 15-16, pp. 1951-1972, 2007.

[26] A. Rajendran, C. J. Magesh, and P. T. Perumal, "DNA-DNA cross-linking mediated by bifunctional [Salen $\left.\mathrm{Al}^{\mathrm{III}}\right]+$ complex," Biochimica et Biophysica Acta. General Subjects, vol. 1780, no. 2, pp. 282-288, 2008.

[27] R. Herchel, Z. Sindelár, Z. Trávnícek, R. Zboril, and J. Vanco, "Novel 1D chain $\mathrm{Fe}(\mathrm{III})$-salen-like complexes involving anionic heterocyclic N-donor ligands. Synthesis, X-ray structure, magnetic, ${ }^{57} \mathrm{Fe}$ Mössbauer, and biological activity studies," Dalton Transactions, 2009.

[28] K. I. Ansari, J. D. Grant, G. A. Woldemariam, S. Kasiri, and S. S. Mandal, "Iron(III)-salen complexes with less DNA cleavage activity exhibit more efficient apoptosis in MCF7 cells," Organic and Biomolecular Chemistry, vol. 7, no. 5, pp. 926-932, 2009. 
[29] K. I. Ansari, S. Kasiri, J. D. Grant, and S. S. Mandal, "Iron (III)salen and salphen complexes induce apoptosis in tumor cells," Journal of Biomolecular Screening, vol. 16, pp. 26-35, 2011.

[30] C. D. Selassie, Burger's Medicinal Chemistry and Drug Discovery, IncCalifornia, 2003.

[31] A. Mohajeri and M. H. Dinpajooh, "Structure-toxicity relationship for aliphatic compounds using quantum topological descriptors," Journal of Molecular Structure, vol. 855, no. 1-3, pp. $1-5,2008$.

[32] K. M. Nikolic, "QSAR study of $\alpha$-tocopherol derivatives with chemotherapeutic activity against human breast cancer cells," Journal of Molecular Structure, vol. 809, no. 1-3, pp. 137-143, 2007.

[33] S. Gao and C. Cao, "Extending bond orbital-connection matrix method to the QSPR study of alkylbenzenes: some thermochemical properties," Journal of Molecular Structure, vol. 778, no. 1-3, pp. 5-13, 2006.

[34] H.-J. Huang, H. W. Yu, C.-Y. Chen et al., "Current developments of computer-aided drug design," Journal of the Taiwan Institute of Chemical Engineers, vol. 41, no. 6, pp. 623-635, 2010.

[35] C. H. Andrade, K. F. M. Pasqualoto, E. I. Ferreira, and A. J. Hopfinger, “4D-QSAR: perspectives in drug design," Molecules, vol. 15, no. 5, pp. 3281-3294, 2010.

[36] D. C. Young, Computational Chemistry, A Practical Guide for Applying-Techniques to Real-Word Problems, Cytoclonal Pharmaceutics Inc, 2001.

[37] T. Fujita, QSAR and Drug Design: New Developments and Applications, Elsevier Science, Amsterdam, The Netherlands, 1995.

[38] T. Puzyn, J. Leszczynski, and M. T. D. Cronin, Recent Advances in QSAR Studies, 2010.

[39] L. M. A. Mullen, P. R. Duchowicz, and E. A. Castro, "QSAR treatment on a new class of triphenylmethyl-containing compounds as potent anticancer agents," Chemometrics and Intelligent Laboratory Systems, vol. 107, no. 2, pp. 269-275, 2011.

[40] C. J. Cramer, Essential of Computational Chemistry Theories and Models, Department of Chemistry and Supercomputing Institute, University of Minnesota, 2002.

[41] “Gaussian 03, Revision C.02," Gaussian Inc., Wallingford, Conn, USA, 2004

[42] R. Todeschini, "Milano Chemometrics and QSAR Group," 2008, http://michem.disat.unimib.it.

[43] U. Sarkar, J. Padmanabhan, R. Parthasarathi, V. Subramanian, and P. K. Chattaraj, "Toxicity analysis of polychlorinated dibenzofurans through global and local electrophilicities," Journal of Molecular Structure, vol. 758, no. 2-3, pp. 119-125, 2006.

[44] R. F. W. Bader, Atoms in Molecules-A Quantum Theory, Clarendon Press, Oxford, UK, 1990.

[45] SPSS Base 10.0 Applications Guide SPSS Inc, Chicago, Ill, USA, 1999.

[46] E. Ibezim, P. R. Duchowicz, E. V. Ortiz, and E. A. Castro, "QSAR on aryl-piperazine derivatives with activity on malaria," Chemometrics and Intelligent Laboratory Systems, vol. 110, no. 1, pp. 81-88, 2012.

[47] K. Kraim, D. Khatmi, Y. Saihi, F. Ferkous, and M. Brahimi, "Quantitative structure activity relationship for the computational prediction of $\alpha$-glucosidase inhibitory," Chemometrics and Intelligent Laboratory Systems, vol. 97, no. 2, pp. 118-126, 2009.

[48] Matlab 7.0, The MathWorks Inc, http://www.mathworks.com.
[49] J. H. A. Al-Fahemi, D. L. Cooper, and N. L. Allan, "QSAR using momentum-space and trivial feature count descriptorsan application to Tetrahymena pyriformis toxicity," Journal of Molecular Structure, vol. 901, no. 1-3, pp. 56-59, 2009.

[50] X.-F. Yan, H.-M. Xiao, X.-D. Gong, and X.-H. Ju, "A comparison of semiempirical and first principle methods for establishing toxicological QSARs of nitroaromatics," Journal of Molecular Structure, vol. 764, no. 1-3, pp. 141-148, 2006.

[51] A. H. Morales, P. R. Duchowicz, M. Á. C. Pérez, E. A. Castro, M. N. D. S. Cordeiro, and M. P. González, "Application of the replacement method as a novel variable selection strategy in QSAR-1. Carcinogenic potential," Chemometrics and Intelligent Laboratory Systems, vol. 81, no. 2, pp. 180-187, 2006.

[52] S. Caetano and Y. Vander Heyden, "Modelling the quality of enantiomeric separations based on molecular descriptors," Chemometrics and Intelligent Laboratory Systems, vol. 84, no. 12, pp. 46-55, 2006.

[53] J. Devillers, Neural Networks in QSAR and Drug Design, CTIS, Lyon, France, 1996.

[54] V. Kovalishyn, J. Aires-de-Sousa, C. Ventura, R. Elvas Leitão, and F. Martins, "QSAR modeling of antitubercular activity of diverse organic compounds," Chemometrics and Intelligent Laboratory Systems, vol. 107, no. 1, pp. 69-74, 2011.

[55] M. Jalali-Heravi, M. Asadollahi-Baboli, and A. ManiVarnosfaderani, "Shuffling multivariate adaptive regression splines and adaptive neuro-fuzzy inference system as tools for QSAR study of SARS inhibitors," Journal of Pharmaceutical and Biomedical Analysis, vol. 50, no. 5, pp. 853-860, 2009.

[56] J. Luo, J. Hu, L. Fu, C. Liu, and X. Jin, "Use of artificial neural network for a QSAR study on neurotrophic activities of N-p-tolyl/phenylsulfonyl L-amino acid thiolester derivatives," Procedia Engineering, vol. 15, pp. 5158-5163, 2011.

[57] O. Deeb, "Correlation ranking and stepwise regression procedures in principal components artificial neural networks modeling with application to predict toxic activity and human serum albumin binding affinity," Chemometrics and Intelligent Laboratory Systems, vol. 104, no. 2, pp. 181-194, 2010.

[58] E. B. de Melo, J. P. A. Martins, T. C. M. Jorge, M. C. Friozi, and M. M. C. Ferreira, "Multivariate QSAR study on the antimutagenic activity of flavonoids against 3-NFA on Salmonella typhimurium TA98," European Journal of Medicinal Chemistry, vol. 45, no. 10, pp. 4562-4569, 2010.

[59] E. Pourbasheer, S. Riahi, M. R. Ganjali, and P. Norouzi, "QSAR study on melanocortin-4 receptors by support vector machine," European Journal of Medicinal Chemistry, vol. 45, no. 3, pp. 1087-1093, 2010.

[60] A. G. Mercader, P. R. Duchowicz, M. A. Sanservino, F. M. Fernández, and E. A. Castro, "QSPR analysis of fluorophilicity for organic compounds," Journal of Fluorine Chemistry, vol. 128, no. 5, pp. 484-492, 2007.

[61] P. R. Duchowicz, E. A. Castro, F. M. Fernández, and M. P. Gonzalez, "A new search algorithm for QSPR/QSAR theories: normal boiling points of some organic molecules," Chemical Physics Letters, vol. 412, no. 4-6, pp. 376-380, 2005.

[62] N.-X. Tan, P. Li, H.-B. Rao, Z.-R. Li, and X.-Y. Li, "Prediction of the acute toxicity of chemical compounds to the fathead minnow by machine learning approaches," Chemometrics and Intelligent Laboratory Systems, vol. 100, no. 1, pp. 66-73, 2010.

[63] Z. Cheng, Y. Zhang, and W. Fu, "QSAR study of carboxylic acid derivatives as HIV-1 Integrase inhibitors," European Journal of Medicinal Chemistry, vol. 45, no. 9, pp. 3970-3980, 2010. 
[64] A. G. Mercader and A. B. Pomilio, "QSAR study of flavonoids and biflavonoids as influenza H1N1 virus neuraminidase inhibitors," European Journal of Medicinal Chemistry, vol. 45, no. 5, pp. 1724-1730, 2010.

[65] P. Paixão, L. F. Gouveia, and J. A. G. Morais, "Prediction of the in vitro intrinsic clearance determined in suspensions of human hepatocytes by using artificial neural networks," European Journal of Pharmaceutical Sciences, vol. 39, no. 5, pp. 310$321,2010$.

[66] M. Goodarzi, P. R. Duchowicz, M. P. Freitas, and F. M. Fernández, "Prediction of the Hildebrand parameter of various solvents using linear and nonlinear approaches," Fluid Phase Equilibria, vol. 293, no. 2, pp. 130-136, 2010. 

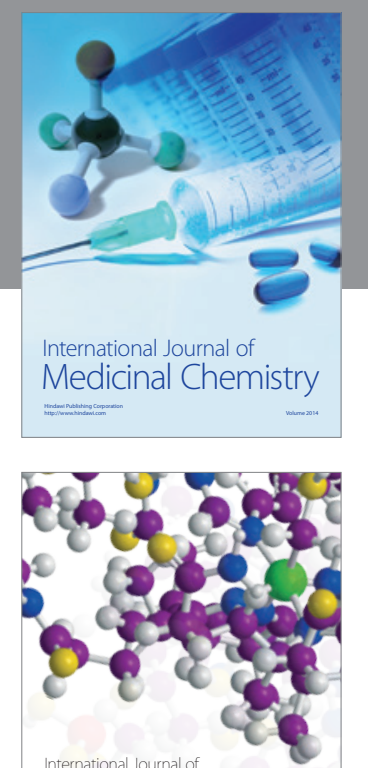

\section{Carbohydrate} Chemistry

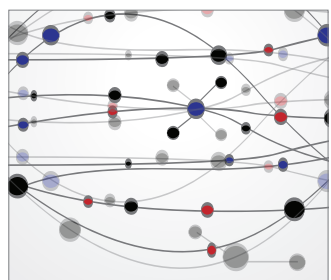

The Scientific World Journal
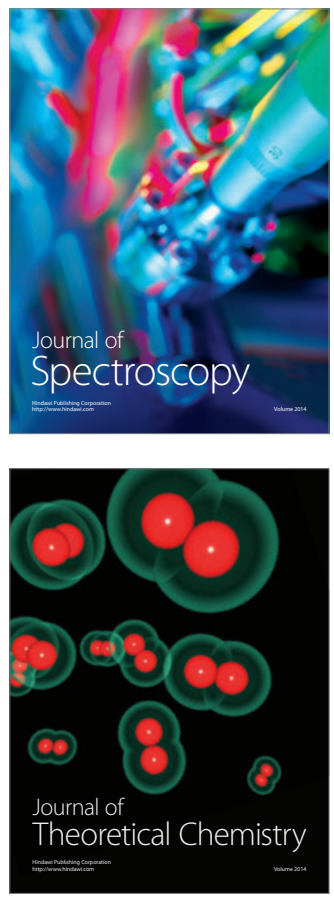
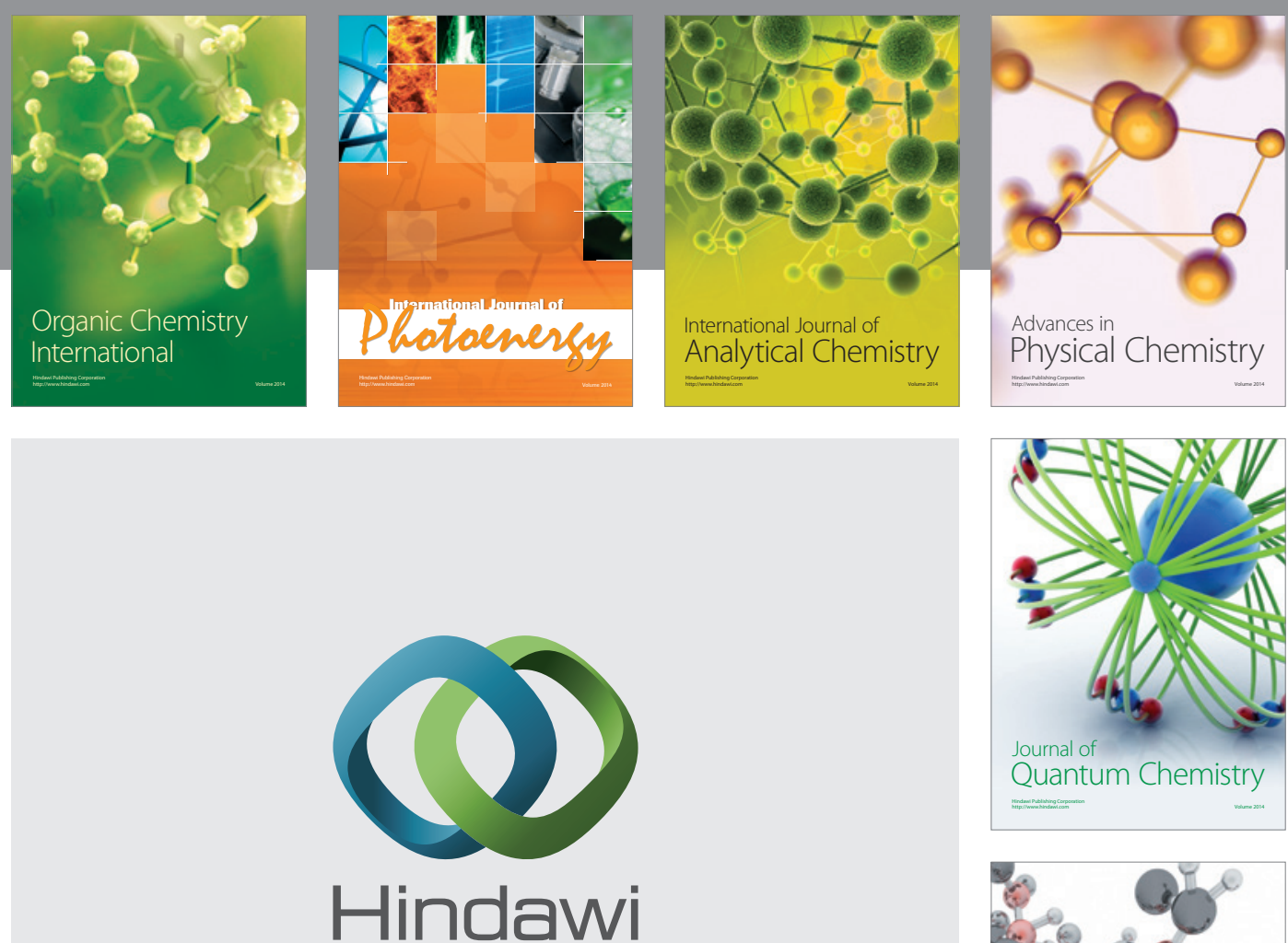

Submit your manuscripts at

http://www.hindawi.com

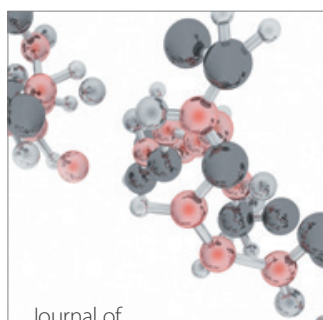

Analytical Methods

in Chemistry

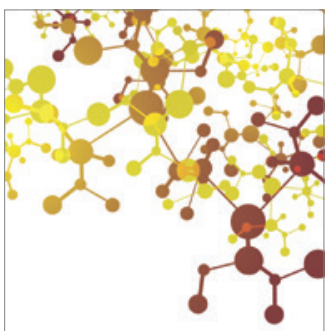

Journal of

Applied Chemistry

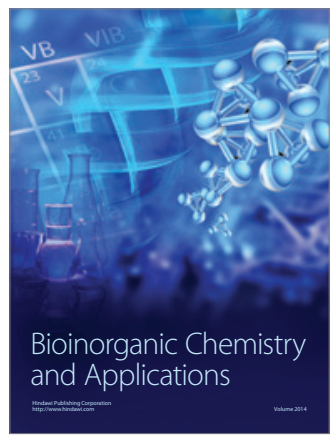

Inorganic Chemistry
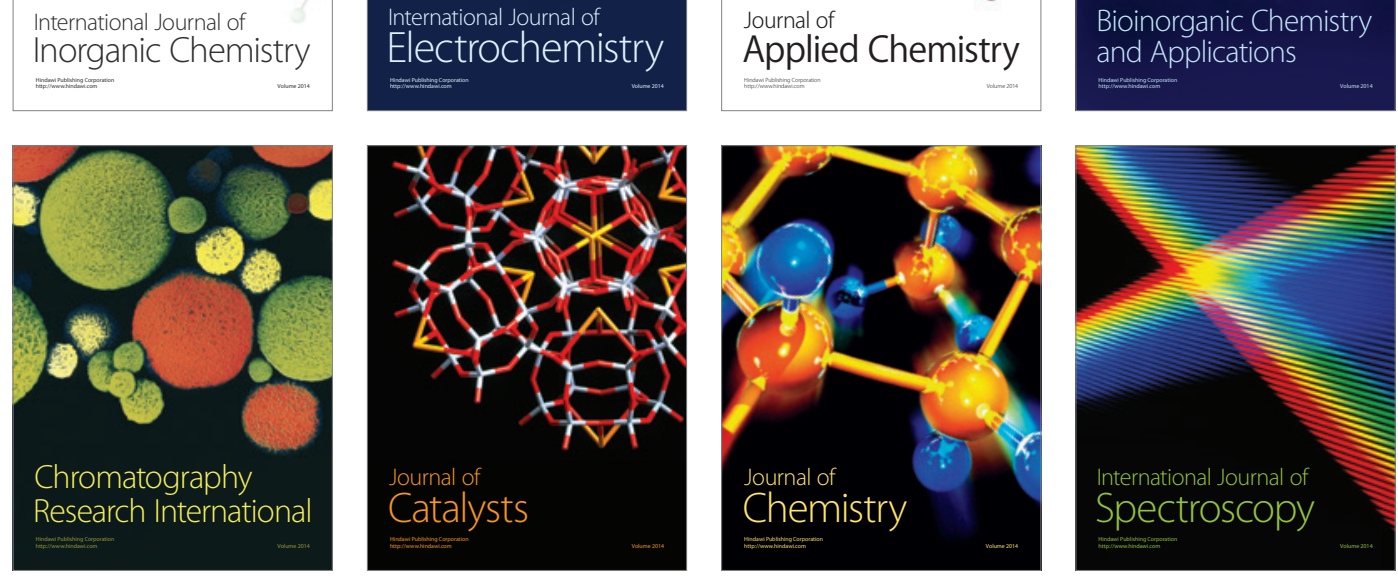